\title{
Unusual case of tubercular peritonitis
}

\author{
L. Thulasi Devi, Athul R. S.*
}

Department of Obstetrics and Gynaecology, Military Hospital, Thiruvananthapuram, Kerala, India

Received: 17 February 2021

Accepted: 15 March 2021

\section{*Correspondence:}

Dr. Athul R. S.

E-mail: athulrajan9@gmail.com

Copyright: () the author(s), publisher and licensee Medip Academy. This is an open-access article distributed under the terms of the Creative Commons Attribution Non-Commercial License, which permits unrestricted non-commercial use, distribution, and reproduction in any medium, provided the original work is properly cited.

\section{ABSTRACT}

An interesting case of Pelvic Tubercular Peritonitis, post MTP and IUCD insertion presenting as Severe Sepsis. Managed aggressively with Fluid, supportive measures and complete course of ATT. Case is being reported as an unusual complication post MTP and incidental reports in literature of spread of Pelvic Peritonitis in preexisting Genital Tuberculosis by haematogenous spread.

Keywords: MTP, IUCD, Pelvic tubercular peritonitis, Severe sepsis

\section{INTRODUCTION}

Female genital tuberculosis (FGTB) is caused by Mycobacterium tuberculosis (rarely Mycobacterium bovis and/or Atypical Mycobacteria) being usually secondary to Tuberculosis (TB) of the lungs or other organs with infection reaching through haematogenous, lymphatic route or direct spread from abdominal $\mathrm{TB}$ or by direct instrumentation. In FGTB, fallopian tubes are affected in $90 \%$ women, whereas uterine endometrium is affected in $70 \%$ and ovaries in about $25 \%$ women. TB remains a major worldwide problem with significant morbidity and mortality. It ranks as the tenth leading cause of death worldwide. ${ }^{1}$ TB is primarily a disease of the lungs, but it can affect almost any organ in the body. Extrapulmonary TB refers to TB involving organs other than the lungs (e.g., pleura, lymph nodes, abdomen, genitourinary tract, skin, joints and bones, or meninges). ${ }^{2}$ Peritoneal TB is a variant of abdominal TB; it poses a public health problem in endemic regions of the world.

The aim of this case report is to describe acute presentation of Pelvic Tuberculosis masquerading as pelvic peritonitis with Septic Shock and to review recent development in the methods of diagnosis and treatment of Pelvic Tuberculosis.

\section{CASE REPORT}

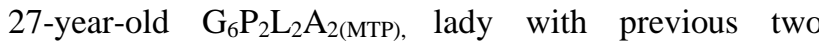
Spontaneous Vaginal Delivery by Midwife without any intrapartum and postpartum complications presented to us This Period of Gestation (POG), 7 weeks and 1 day with single Live Intrauterine gestation with Fetal cardiac activity. She requested for MTP with IUCD. Her GPE was within normal limits. Systemic Examination was unremarkable. She had no comorbidities and no past history of any major illness or Koch's. She denied any history of previous gynaecological instrumentation or manipulation.

She was administered Mifepristone 600mg PO and Misoprostol $800 \mu \mathrm{g}$ sublingually after $48 \mathrm{hrs}$. She was taken up for check curettage and IUCD insertion under Anaesthesia (on request of patient) and procedure was carried out under Spinal Anaesthesia (Saddle block). Intraoperatively cervix was negotiated with difficulty with dilators, suggestive of Ashermann's, CuT 375A was inserted under strict asepsis after check curettage (blunt). Immediate Post-operative period was uneventful.

She developed severe irritability, incoherent behaviour, restlessness and air hunger at around $1730 \mathrm{hrs}$ on the day of procedure itself. Immediate examination revealed, 
Tachycardia (128/min), hypotension $\quad(60 / 40 \mathrm{mmHg}$, Preoperative was $90 / 60 \mathrm{~mm} \mathrm{Hg}$ ), dehydration (high coloured urine, no haematuria, with hourly urine output of $20 \mathrm{ml} / \mathrm{hr}$ ), all peripheral pulses were present and bounding with mild fever $\left(100.8^{\circ} \mathrm{F}\right)$, tachypnoea $(26 / \mathrm{mt})$, fall in SPo2 ( $88 \%$ on room air), with no pallor or cyanosis. Abdominal Examination revealed tenderness over suprapubic region, with minimal guarding and rigidity, Internal examination revealed tenderness on rocking of cervix, with boggy swelling and tenderness in POD and b/l adnexa. IUCD was in situ. Grey Turners' and Kehr's sign was negative. Other Systemic Examination was unremarkable.

Investigations revealed Normocytic, Normochromic Anaemia with leucocytosis, shift to left and presence of toxic granules. All other biochemical and haematological parameters were within normal limits and she was seronegative for HIV. Skiagram of chest was normal with no air under the diaphragm or blunting of CP angles. Radiological examination (TVS) revealed collection in anterior pouch, POD, bilateral fornices, uterine cavity, with IUCD in situ (Figure 1 and 2).

TAS did not reveal any other pathology, Morrison's pouch and subhepatic space did not show any collection. Simultaneous aggressive resuscitation with colloids, third generation parenteral antibiotics and ionotropes was done. A working diagnosis of Septic Shock was made, and patient managed accordingly. Surgical complications of MTP i.e. Perforation and Haemorrhagic Shock were kept in differential diagnosis as the presentation was acute and within six hours of completion of procedure. Drug reaction and anaphylaxis was ruled out and opinion of intensivist was taken. Blood was kept on standby.

On $1^{\text {st }}$ postoperative day she had persistent pain and tenderness, IUCD was removed, endometrial collection which was straw coloured was drained and sent for culture, biochemistry, cytology and AFB (sterile after $48 \mathrm{hrs,}$ lymphocyte predominant, no AFB seen). Percutaneous trans abdominal drainage of straw coloured ascitic fluid was done and sent for cytology, biochemistry, culture, SAAG ratio, TB-PCR \& AFB (sterile after $48 \mathrm{hrs,}$ lymphocyte predominant, no AFB seen and SAAG was suggestive of exudative peritonitis). Her vitals were stable although abdominal signs persisted. She was continued on antibiotics as the results of above tests were awaited. However, a strong clinical suspicion of Acute Pelvic Tubercular peritonitis was now considered and haemorrhagic shock was ruled out. TVS revealed ascites, endometrial collection (tubercular endometritis) with numerous thin complete septations and fine, delicate, incomplete and mobile strands of fibrin deposits, partially particulated and partially loculated in some areas which is hallmark of "WET TUBERCULOSIS" as referred previously (Figure 3 and 4).

CT and MRI were not available at the centre and patient refused transfer due to COVID lockdown situation and she showed subjective improvement. On $2^{\text {nd }}$ postoperative day patient continued to complain of suprapubic tenderness and fullness. Her GPE and Systemic examination was unremarkable however patient looked "sick and unwell". ATT (EHRZ) was started based on strong clinical suspicion with available biochemical parameters (TB-PCR was awaited) and radiological findings. Parenteral antibiotics were continued. She was ambulant and was accepting oral fluids.

On $3^{\text {rd }}$ postoperative day TB- PCR was reported positive (LJ culture was not available). Parenteral antibiotics were discontinued and ATT was continued.

On $4^{\text {th }}$ postoperative day patient was ambulant, cheerful, talkative and showed remarkable improvement. Nursing Officers reported adequate and satisfactory oral intake.

On $5^{\text {th }}$ postoperative day she requested for discharge and expressed desire to meet her children. She was counselled and was advised to wait till $7^{\text {th }}$ postoperative day due to her stormy course and unusual presentation. TVS was repeated which showed resolution of Endometritis with minimal ascites (Figure 5 and 6).

On $7^{\text {th }}$ postoperative day a detailed summary was given to her and she was counselled to complete ATT as advised (2EHRZ+4HR) and was discharged with advice to follow up after 15 days or SOS.

On $15^{\text {th }}$ day of follow up she was asymptomatic and all her biochemical and haematological parameters were within normal limits. TVS was repeated and it showed resolving endometritis and peritonitis (Figure 7 and 8). She continued ATT for 6 months and was on regular follow up and was asymptomatic. All biochemical and haematological parameters were within normal limits.

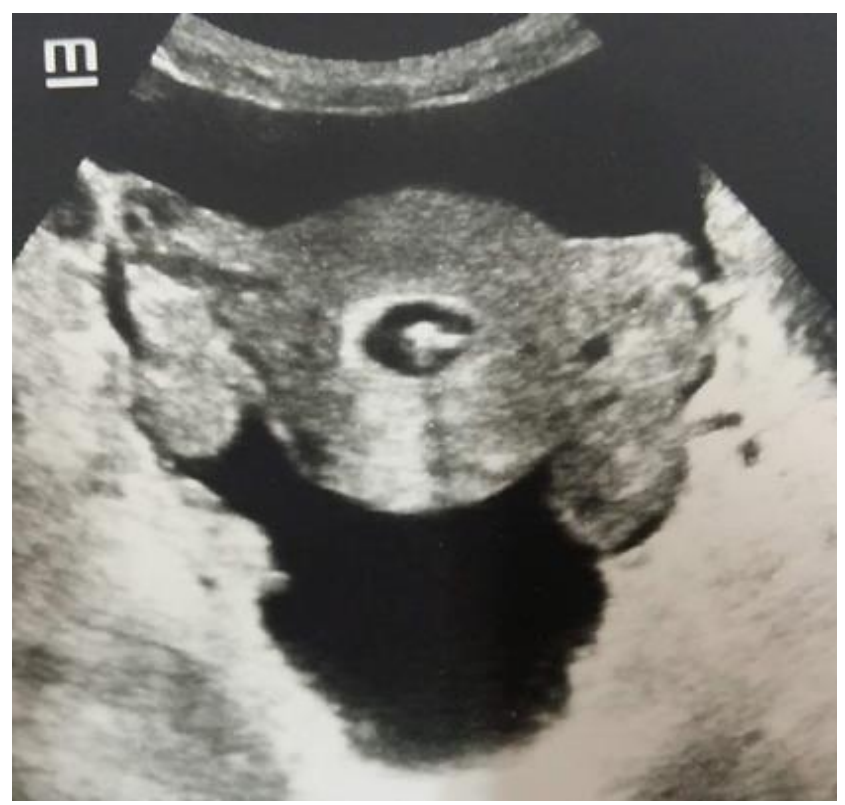

Figure 1: (TVS) Reveals collection in anterior pouch, POD, bilateral fornices and endometrial cavity with IUCD in situ. 


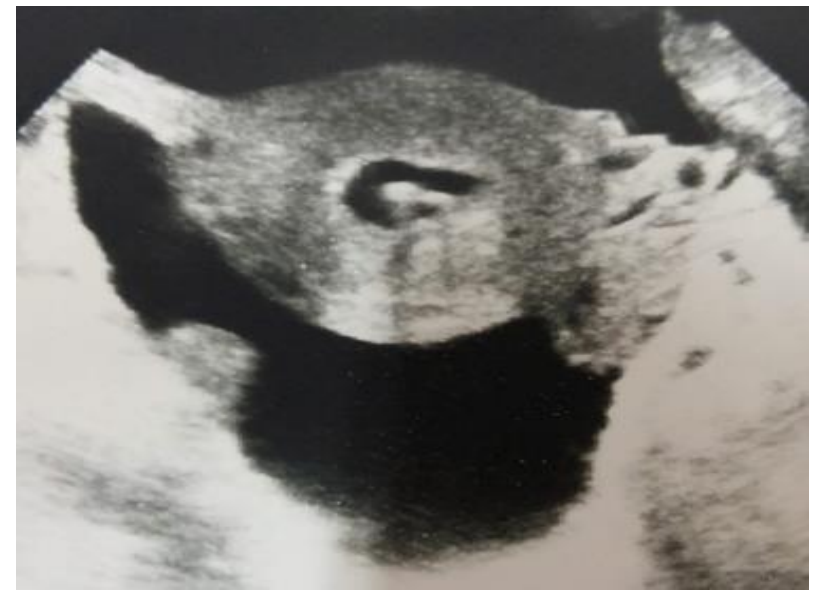

Figure 2: (TVS) Reveals collection in anterior pouch, POD, bilateral fornices and endometrial cavity with IUCD in situ.

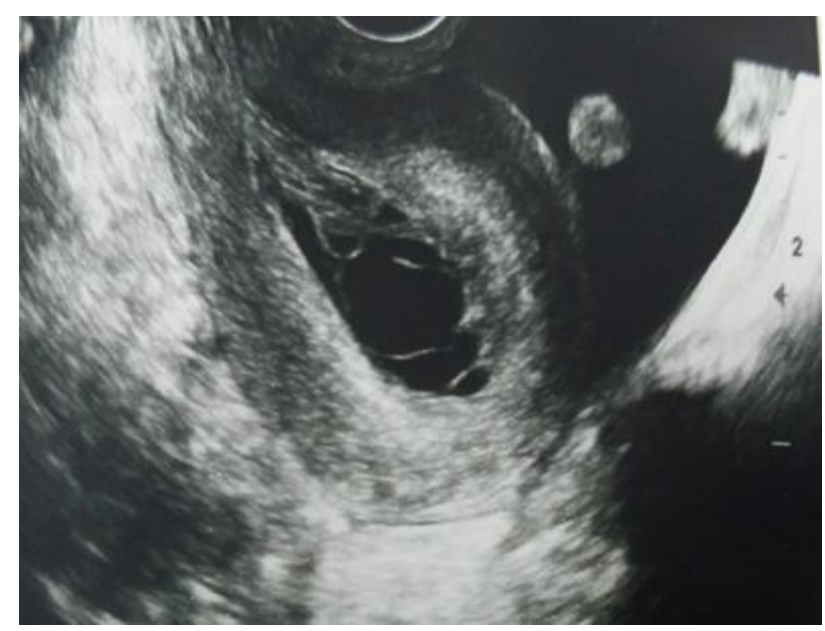

Figure 3: (TVS) of fibrin collection in endometrial cavity with thin septations and loculations with ascitic fluid. (after IUCD removal).

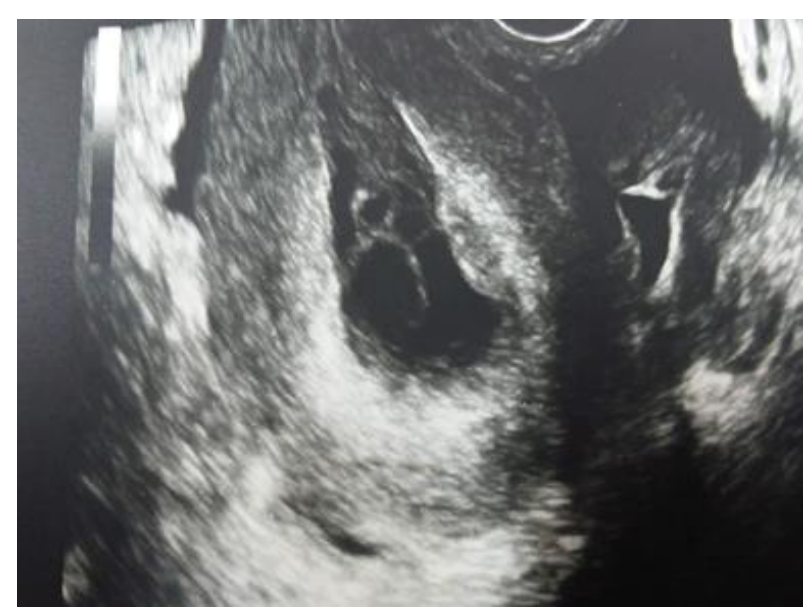

Figure 4: (TVS) of fibrin collection in endometrial cavity with thin septations and loculations with particulated ascitic fluid. (after IUCD removal).

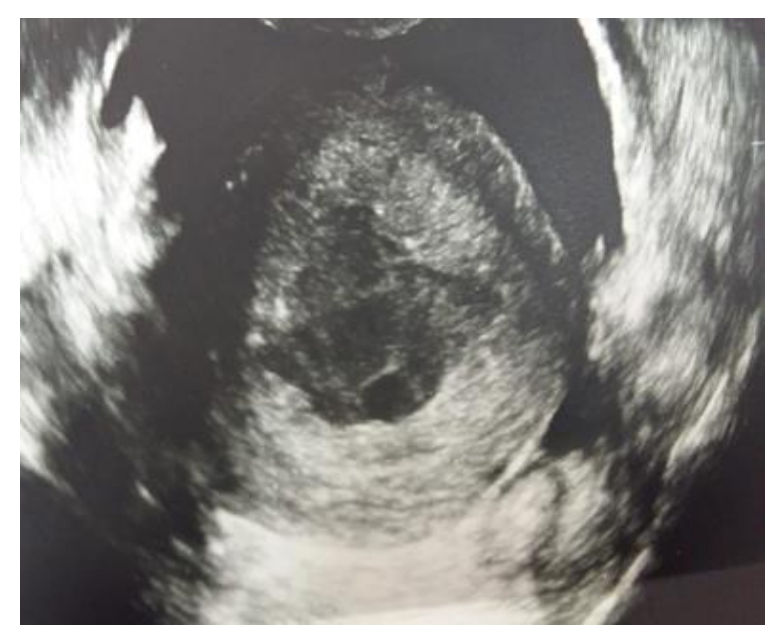

Figure 5: (TVS) Partial resolution of endometritis, loculations and fibrin strands with minimal ascites.

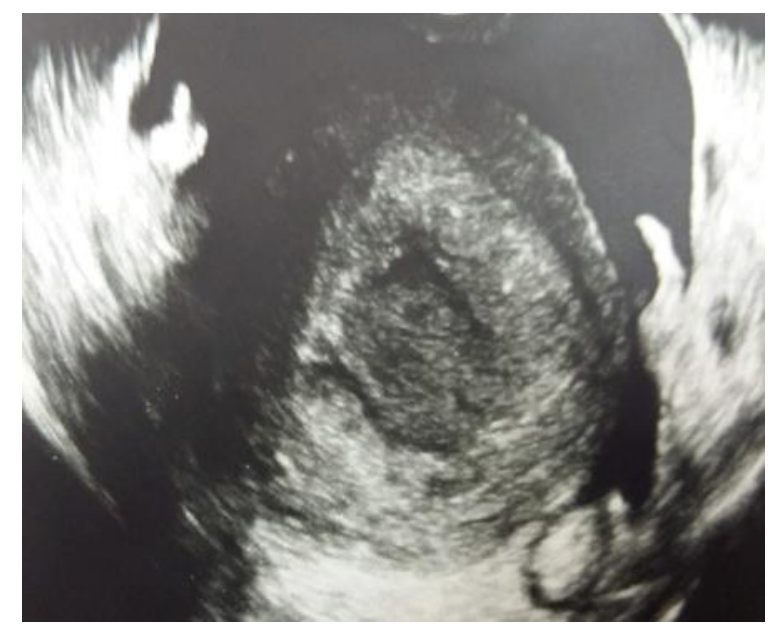

Figure 6: (TVS) Partial resolution of endometritis, loculations and fibrin strands with minimal ascites.

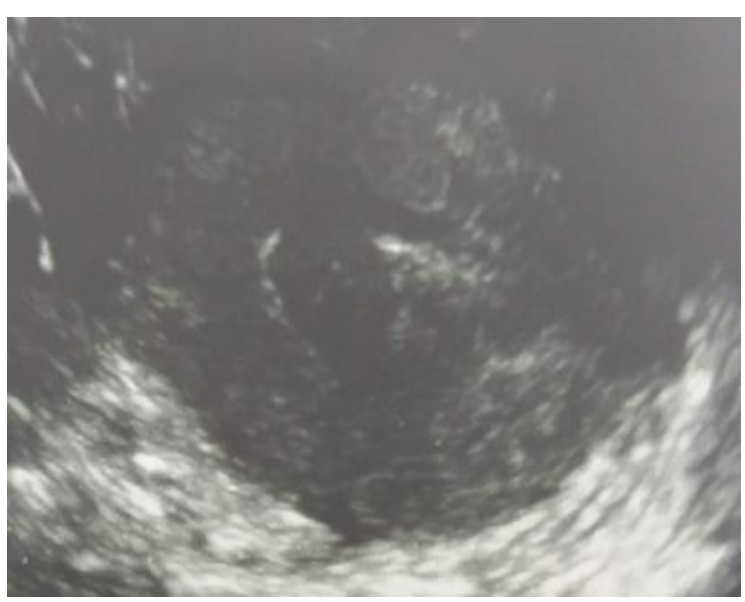

Figure 7: (TVS) Resolving endometritis and peritonitis. 


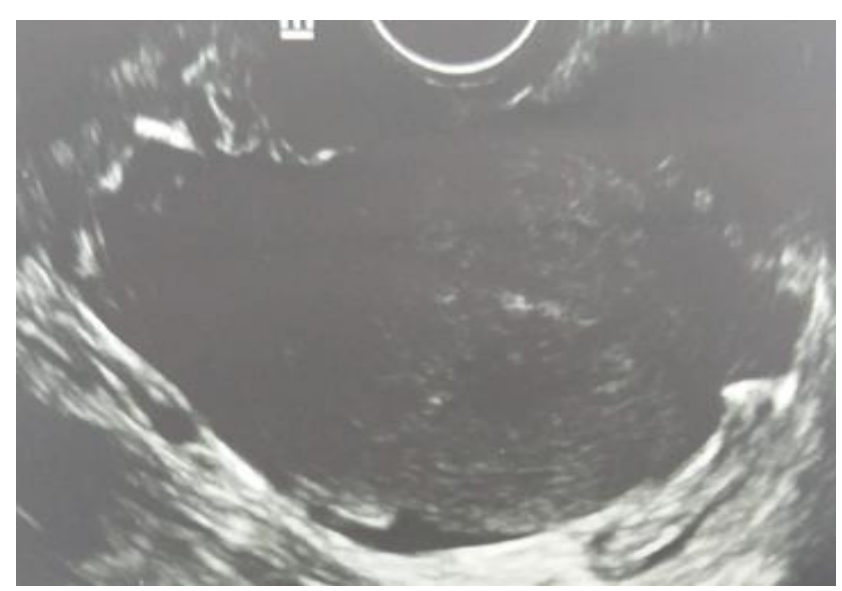

Figure 8: (TVS) Resolving endometritis and peritonitis.

\section{DISCUSSION}

TB is a worldwide disease. In 2015, there were an estimated 10.4 million new TB cases worldwide, of which 5.9 million (56\%) were men and 3.5 million (34\%) were women. Children accounted for 1.0 million (10\%), and people living with HIV accounted for 1.2 million (11\%) of all new TB cases. Moreover, there were an estimated 480,000 new cases of multidrug-resistant $\mathrm{TB} .{ }^{1} \mathrm{~TB}$ has strong associations with socioeconomic factors such as poverty, malnutrition, deprivation, overcrowding, illiteracy and limited access to health-care facilities, having a high prevalence in developing countries. ${ }^{3}$ Peritoneal TB accounts for about $0.1 \%-0.7 \%$ of all cases of TB representing $4 \%-10 \%$ of extra-pulmonary TB and $25 \%-60 \%$ of abdominal TB cases. ${ }^{4-6}$ Although peritoneal TB can develop at any age, peritoneal TB is seen more commonly between 25 and 55 years of age. The gender difference among patients with peritoneal TB varies from study to study and from country to another. In general, in developing countries, ${ }^{7-11}$ the disease is observed predominantly in females, while in industrialized and some rich countries, there is male predominance which may be attributed to the presence of male immigrants in the workforce. ${ }^{6,12-15}$

The postulated mechanisms by which peritoneum can be involved are: ${ }^{16,17}$

1) Reactivation of latent tuberculous foci in the peritoneum, acquired by haematogenous spread from a primary lung focus. 2) Haematogenous spread from active pulmonary, miliary TB or silent bacteremia during the primary phase of TB. 3) Direct spread from infected organs such as intestine, fallopian tubes and rupture of a tuberculous intra-abdominal lymph node. 4) Through lymph channels from infected abdominal lymph nodes. Abdominal lymph nodal and peritoneal TB may occur without gastrointestinal involvement.

Peritoneal TB occurs in three forms: 1) Wet type with ascites, 2) Encysted (loculated) type with a localized abdominal swelling, and 3) Fibrotic type with abdominal masses composed of mesenteric and omental thickening, with matted bowel loops, felt as lumps in the abdomen. Considerable overlap of these types can be observed. ${ }^{6}$

The typical analysis of ascitic fluid from patients with peritoneal TB demonstrates high protein concentration $(>3$ $\mathrm{g} / \mathrm{dl}$ ) with serum to ascites albumin gradient of $<1.1 \mathrm{~g} / \mathrm{dl}$ and predominant lymphocytic pleocytosis. Identification of Acid-fast bacilli in the ascitic fluid through both smear and culture methods remains a useful mean to diagnose peritoneal TB. Techniques to improve the diagnostic yield of TB culture in the peritoneal fluid were attempted. Singh et al. assumed that $1 \mathrm{~L}$ of ascitic fluid can provide upto $83 \%$ of positive results. ${ }^{16}$ This technique is impractical as this large amount of fluid needs special centrifuge machines. Interestingly, conventional mycobacterial culture takes up to 4-6 weeks to achieve results, even with liquid culture methods the process requires at least 12 days. This delay may increase morbidity and therefore, other diagnostic tools for early diagnosis of TB are needed. The performance of conventional polymerase chain reaction (PCR) was disappointing. Abdominal sonography is the most commonly used first-line imaging modality for evaluating patients with suspected peritoneal TB as it can easily assess the peritoneum. ${ }^{18}$ The most common findings include multiple thin septae, visible debris of different densities within the fluid. Other findings include peritoneal thickening and nodularity. ${ }^{18-20}$ As illustrated in many reports, these findings lack specificity and are not useful for differentiating peritoneal TB from other diseases such as peritoneal carcinomatosis. ${ }^{6,18}$ However, abdomen Sonography can be used to guide abdomen tapping and peritoneal biopsy. Peritoneal biopsy gives a better diagnostic value than ascitic fluid alone. It can be obtained by different procedures including blind percutaneous peritoneal biopsy, Transabdominal sonographic, or CT-guided peritoneal biopsy and laparotomy or laparoscopic biopsy. Characteristic laparoscopic appearance includes free ascites, multiple yellowish-white nodules or tubercles on visceral and parietal peritoneum, peritoneal or visceral adhesion, and occasionally inflamed haemorrhagic areas on the peritoneum. It was reported that macroscopic picture of peritoneum during laparoscopy was suggestive of TB in $85 \%-100 \%$ of the cases and laparoscopically guided peritoneal biopsy had detected caseating granulomas in $76 \%-100 \%$ of patients. Microbiological studies of biopsy should be performed in all cases to identify nontuberculous mycobacteria (especially in patients on continuous ambulatory peritoneal dialysis), to detect drug resistance $\mathrm{TB}$ and to increase the diagnostic yield of the procedure, as it may be positive even in the absence of a characteristic histopathological picture. Laparoscopic biopsy specimens reveal Acid-fast bacilli in 3\%-25\% and cultures were positive for M. tuberculosis in 38\%-98\%. There is lack of data on diagnostic yield of PCR study for M. tuberculosis on peritoneal biopsy. Although laparoscopic biopsy is safe, it is not free of complications such as intestinal perforation and haemorrhage., ${ }^{61-24}$ 
Laparoscopy was deferred due to clinical condition of the patient and prevalence of the disease with available biochemical and pathological correlation. She showed remarkable improvement after a fortnight of ATT.

\section{CONCLUSION}

Available data show that there is no specific sign or symptom for the diagnosis of peritoneal TB. A high index of suspicion is always required. Peritoneal TB should be considered early as a differential diagnosis in patients who have unexplained low gradient ascites with high lymphocytes. The role of advanced diagnostic modalities such as modified PCR techniques and biomarkers remain controversial. Laparoscopically guided peritoneal biopsy remains the best diagnostic tool in our hand nowadays as it provides a rapid and correct diagnosis, therefore, it should be performed early in suspected cases. Six months of treatment with the 4-drug regimen is effective and markedly improves the outcome.

The currently recommended regimen for treatment of peritoneal TB is largely similar to treatment for TB elsewhere and includes the "4-drug regimen;" an initial phase of 2 months followed by a continuation phase of 4 months. Treatment in the 2-month phase is usually including daily administration of rifampicin, isoniazid; pyrazinamide and ethambutol or streptomycin. If the isolate is susceptible to rifampicin and isoniazid, pyrazinamide, and ethambutol can be discontinued followed by a continuation phase where isoniazid and rifampicin are again given daily for 4 months. Some physicians use antituberculous therapy for 9-12 months without any scientific justification. The optimal duration of therapy is debatable.

Some physicians use antituberculous therapy for 9-12 months without any scientific justification. In our experience, anti-TB drug therapy can be used for 9 months or 12 months in a patient who uses second-line therapy due to drug resistance or drug sensitivity. Duration of therapy also can be extended because of side effect developed during treatment. Corticosteroid administration combined with anti-TB treatment has been advocated by some researchers to reduce the complications; however, there is a controversy about the benefit that can be obtained. We did not consider it appropriate to exhibit steroid in our patient.

Funding: No funding sources Conflict of interest: None declared

Ethical approval: Not required

\section{REFERENCES}

1. WHO. Global Tuberculosis; 2016. Available at: http://www.who. int/ tb/publications/global_report /en/. Accessed on $15^{\text {th }}$ February 2021.
2. Lee JY. Diagnosis and treatment of extrapulmonary tuberculosis. Tuberc Respir Dis (Seoul) 2015;78:4755.

3. Prevention and control programs. Enferm Infec Microbiol Clin. 2011;29 Suppl 1:2-7.

4. Sanai FM, Bzeizi KI. Systematic review: Tuberculousperitonitis - Presenting features, diagnostic strategies and treatment.Aliment Pharmacol Ther. 2005;22:685-700.

5. Kocaman O, Danalioğlu A, İnce AT, Tozlu M, Şentürk H. Diagnosis of tuberculous peritonitis using endoscopic ultrasound-guided fine-needle aspiration biopsy of the peritoneum. Turk J Gastroenterol. 2013;24:65-9.

6. Khan FY, Al-Muzrakchi AM, Elbedawi MM, AlMuzrakchi AA, Al Tabeb A. Peritoneal tuberculosis in Qatar: A five-year hospital-based study from 2005 to 2009. Travel Med Infect Dis. 2012;10:25-31.

7. Manohar A, Simjee AE, Haffejee AA, Pettengell KE. Symptoms and investigative findings in 145 patients with tuberculous peritonitis diagnosed by peritoneoscopy and biopsy over a five year period. Gut. 1990;31:1130-2.

8. El Abkari M, Benajah DA, Aqodad N, Bennouna S, Oudghiri B, Ibrahimi A, et al. Peritoneal tuberculosis in the Fes University Hospital (Morocco). Report of 123 cases. Gastroenterol Clin Biol. 2006;30:377-81.

9. Sattar Z, Iqbal M, Gondal KM. The frequency of peritoneal tuberculosisusing diagnostic laparoscopy in clinically suspected cases. Ann King Edward Med Univ. 2014;20:176-81.

10. Makhlouf NA, Makhlouf GA, Soliman A, Sherif MF, Rashed HA. Diagnostic challenges of tuberculosis peritonitis in upper Egypt. Al Azhar Assiut Med J. 2016;14:115-21.

11. 1Dülger AC, Karadaş S, Mete R, Türkdoğan MK, Demirkıran D, Gültepe B, et al. Analysis of cases with tuberculous peritonitis: A single-center experience. Turk J Gastroenterol 2014;25:72-8.

12. 12. Thoreau N, Fain O, Babinet P, Lortholary O, Robineau M, Valeyre D, et al. Peritoneal tuberculosis: 27 cases in the suburbs of Northeastern Paris. Int $\mathbf{J}$ Tuberc Lung Dis. 2002;6:253-8.

13. Cavalli Z, Ader F, Valour F, Saison J, Boussel L, Dumitrescu O, et al. Clinical presentation, diagnosis, and bacterial epidemiology of peritoneal tuberculosis in two university hospitals in France. Infect Dis Ther. 2016;5:193-9.

14. Chau TN, Leung VK, Wong S, Law ST, Chan WH, Luk IS, et al. Diagnostic challenges of tuberculosis peritonitis in patients with and without end-stage renal failure. Clin Infect Dis 2007;45:141-6.

15. Vaid U, Kane GC. Tuberculous peritonitis. Microbiol Spectr. 2017;5.

16. Singh MM, Bhargava AN, Jain KP. Tuberculous peritonitis. An evaluation of pathogenetic mechanisms, diagnostic procedures and therapeutic measures. N Engl J Med. 1969;281:1091-4.

17. Sharma MP, Bhatia V. Abdominal tuberculosis. Indian J Med Res. 2004;120:305-15. 
18. Hanbidge AE, Lynch D, Wilson SR. US of the peritoneum. Radiographics. 2003;23:663-84.

19. Tongsong T, Sukpan K, Wanapirak C, Sirichotiyakul S, Tongprasert F. Sonographic features of female pelvic tuberculous peritonitis. J Ultrasound Med. 2007;26:77-82.

20. Akhan O, Demirkazik FB, Demirkazik A, Gülekon N, Eryilmaz M, Unsal M, et al. Tuberculous peritonitis: Ultrasonic diagnosis. J Clin Ultrasound 1990;18:711-4.

21. Chow KM, Chow VC, Szeto CC. Indication for peritoneal biopsy in tuberculous peritonitis. Am J Surg 2003;185:567-73.

22. Abid H, Charif I, Mellouki I, Aqodad D, Benajah A, Ibrahim A, et al. Role of laparoscopy in diagnosis of peritoneal tuberculosis (About 414cases). Open J Gastroenterol. 2013;3:205-7.

23. Abdelaal A, Alfkey R, Abdelaziem S, Abunada M, Alfaky A, Ibrahim WH, et al. Role of laparoscopic peritoneal biopsy in the diagnosis of peritoneal tuberculosis. A seven year experience. Chirurgia (Bucur) 2014; 109:330 4.

24. Saxena P, Saxena S. The role of laparoscopy in diagnosis of abdominal tuberculosis. Int Surg J 2016; 3:1557-63.

Cite this article as: Devi LT, Athul RS. Unusual case of tubercular peritonitis Int J Reprod Contracept Obstet Gynecol 2021;10:1707-12. 